\title{
Daily estimation of the severity of multiple organ dysfunction syndrome in critically ill children
}

\author{
Stéphane Leteurtre MD, Alain Duhamel PhD, Bruno Grandbastien MD, François Proulx MD, \\ Jacques Cotting MD, Ronald Gottesman MD, Ari Joffe MD, Bendicht Wagner MD, \\ Philippe Hubert MD, Alain Martinot MD, Jacques Lacroix MD, Francis Leclerc MD
}

Previously published at www.cmaj.ca

$\infty \quad$ See related commentary by Kneyber, page 1155

\section{ABSTRACT}

Background: Daily evaluation of multiple organ dysfunction syndrome has been performed in critically ill adults. We evaluated the clinical course of multiple organ dysfunction over time in critically ill children using the Pediatric Logistic Organ Dysfunction (PELOD) score and determined the optimal days for measuring scores.

Methods: We prospectively measured daily PELOD scores and calculated the change in scores over time for 1806 consecutive patients admitted to seven pediatric intensive care units (PICUs) between September 1998 and February 2000. To study the relationship between daily scores and mortality in the PICU, we evaluated changes in daily scores during the first four days; the mean rate of change in scores during the entire PICU stay between survivors and nonsurvivors; and Cox survival analyses using a change in PELOD score as a time-dependent covariate to determine the optimal days for measuring daily scores.

Results: The overall mortality among the 1806 patients was $6.4 \%$. A high PELOD score ( $\geq 20$ points) on day 1 was associated with an odds ratio (OR) for death of 40.7 (95\% confidence interval $[\mathrm{Cl}] 20.3-81.4)$; a medium score (10-19 points) on day 1 was associated with an OR for death of 4.2 (95\% Cl 2.0-8.7). Mortality was 50\% when a high score on day 1 increased on day 2 . The course of daily PELOD scores differed between survivors and nonsurvivors. A set of seven days (days 1, 2, 5, 8, 12, 16 and 18) was identified as the optimal period for measurement of daily PELOD scores.

Interpretation: PELOD scores indicating a worsening condition or no improvement over time were indicators of a poor prognosis in the PICU. A set of seven days for measurement of the PELOD score during the PICU stay provided optimal information on the progression of multipleorgan dysfunction syndrome in critically ill children.

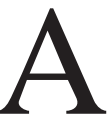
lmost all patients in intensive care units (ICUs) have some organ dysfunction..$^{1-4}$ Adult and pediatric studies have shown that mortality increases with the number of organs involved. ${ }^{2.45}$ Thus, multiple-organ dysfunction syndrome (dysfunction involving two or more organs) has been viewed as the inexorable pathway to death. ${ }^{6}$ Primary multiple-organ dysfunction syndrome (present at admission or occurring within the first week after admission to the ICU) accounts for $88 \%$ of children with the syndrome; secondary multiple-organ dysfunction syndrome is less common (12\%) but is associated with higher morbidity and mortality.

Organ dysfunction scores were first developed for use in critically ill adults to describe and quantify the severity of organ dysfunction, not to predict mortality. Two scores have been proposed for critically ill children: the Pediatric Logistic Organ Dysfunction (PELOD) score and the Pediatric Multiple Organ Dysfunction Score (P-MODS). ${ }^{8-10}$ These scores quantify organ dysfunction precisely and can be used as indicators of the severity of illness throughout the clinical course. They can also be used as baseline and outcome measures in clinical studies conducted in ICUs ${ }^{11,12}$ and pediatric ICUs (PICUs). ${ }^{13}$

The PELOD score calculated with data collected over the entire PICU stay has been validated (using the most abnormal value of each variable during the entire PICU stay). ${ }^{10}$ However, the PELOD score over the entire PICU stay cannot be calculated before discharge from the unit; therefore, it cannot be used to characterize and follow the severity of organ dysfunction on a daily basis. Measurements repeated daily may provide more useful information. ${ }^{14}$ The optimal period for measuring daily scores for multiple organ dysfunction in adults has been studied. ${ }^{15-17}$ Indeed, trends in the Sequential Organ Failure Assessment score over the first 48 hours in the ICU was found to be a sensitive indicator of outcome, with decreasing scores associated with a decrease in mortality from $50 \%$ to $27 \% .{ }^{17}$ Similar data for critically ill children are lacking.

From the Pediatric Intensive Care Unit (Leteurtre, Martinot, Leclerc), the Department of Biostatistics (Duhamel) and the Department of Epidemiology and Public Health (Grandbastien), Université Lille Nord de France, UDSL, EA 2694, Centre hospitalier universitaire Lille, Lille, France; the Pediatric Intensive Care Unit (Proulx, Lacroix), Sainte-Justine Hospital, Montréal, Que.; the Pediatric Intensive Care Unit (Cotting), Centre hospitalier Vaudois, Lausanne, Switzerland; the Pediatric Intensive Care Unit (Gottesman), Montréa Children's Hospital, Montréal, Que.; the Pediatric Intensive Care Unit (Joffe), Stollery Children's Hospital, Edmonton, Alta.; the Paediatric Intensive Care Unit (Wagner), Inselspital, Bern, Switzerland; and the Pediatric Intensive Care Unit (Hubert), Enfants Malades Hospital, Paris, France

CMAJ 2010. DOI:10.1503/cmaj.081715 
We conducted this study to describe the clinical course of multiple organ dysfunction over time as measured by the daily PELOD score. Because the time and effort necessary to ensure accurate daily assessments and data entry can be substantial ${ }^{18}$ we also aimed to determine the optimal days for measuring daily scores during the PICU stay.

\section{Methods}

We included all 2021 consecutive patients admitted to seven multidisciplinary, tertiary care PICUs of university-affiliated hospitals (three in Canada, two in France and two in Switzer-

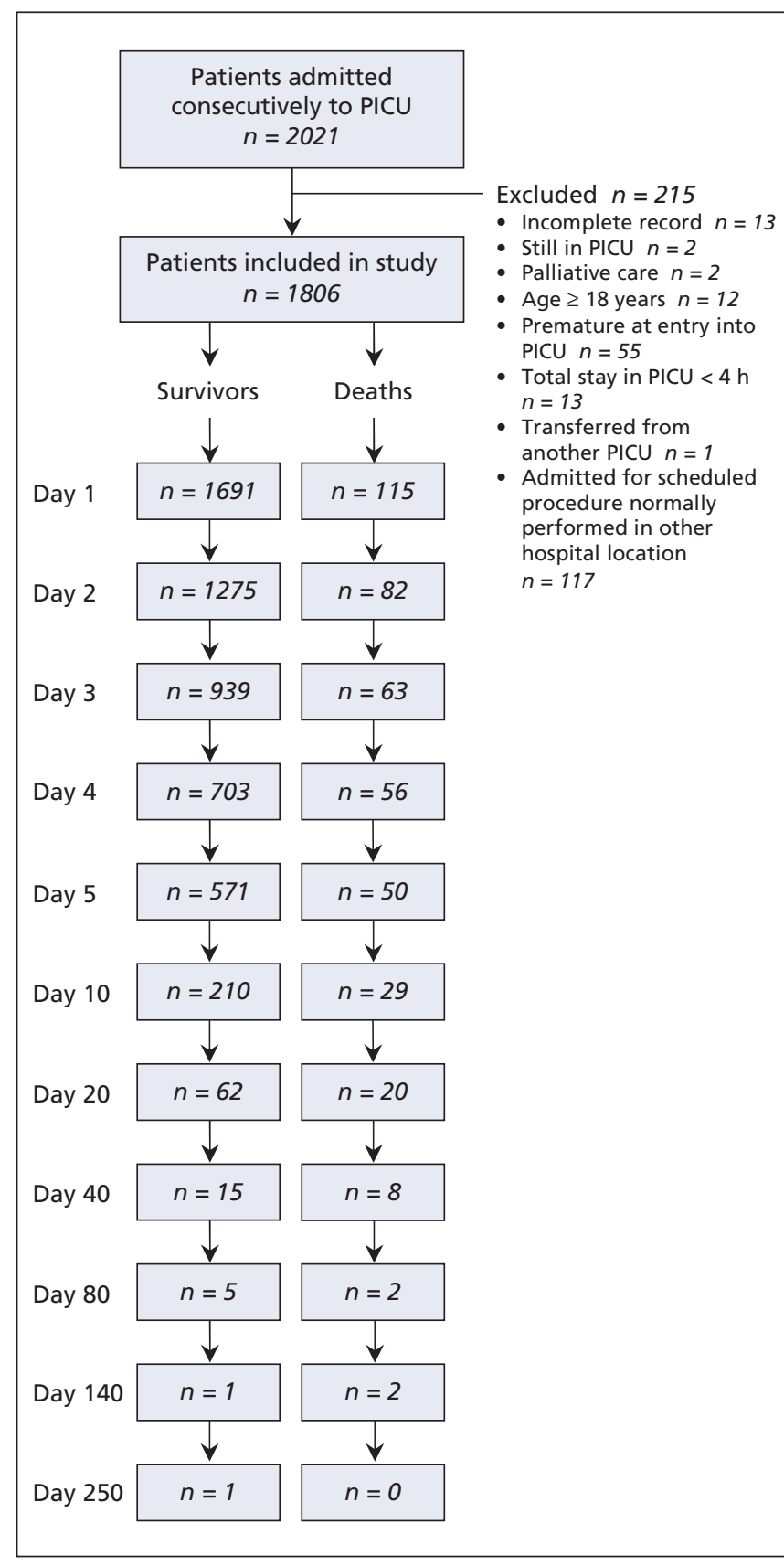

Figure 1: Selection of critically ill children for the study population. $\mathrm{PICU}=$ pediatric intensive care unit. land) between September 1998 and February 2000. We excluded 215 patients for reasons described in Figure 1. The ethics committee of each participating hospital approved the study design.

We collected data on the remaining 1806 patients' baseline characteristics and their length of stay in the PICU. We calculated daily PELOD scores. For the PELOD score, six organ systems (neurologic, cardiovascular, renal, respiratory, hematologic and hepatic) are considered, each with up to 3 variables (total 12 variables). Each variable is assigned points $(0,1,10$ or 20$)$ based on the level of severity (Figure 2). Levels of severity and relative weights of each organ dysfunction were determined by means of logistic regression. ${ }^{10}$ For each variable, the most abnormal value of each day was used to calculate the daily PELOD score. Variables were measured only if requested by the attending physician; if a variable was not measured, it was assumed to be identical to the previous measurement or normal. ${ }^{10}$

The primary outcome was the patient's vital status at discharge from the PICU.

\section{Statistical analysis}

Results are expressed as frequencies and percentages for categorical variables and as medians and interquartile ranges (IQRs) for numeric variables. A $p$ value of less than 0.05 was considered statistically significant.

We investigated the relationship between the PELOD score on day 1 and outcome (survival v. death) using logistic regression analysis. Thereafter, we used three analytical strategies to study the relationship between daily PELOD scores and mortality: (a) changes in daily PELOD scores during the first four days; (b) the mean rate of change in PELOD scores during the entire PICU stay between survivors and nonsurvivors; and (c) survival analyses (Cox model), with the change in daily PELOD score from day 1 as a time-dependent covariate, to determine the optimal period for measuring daily PELOD scores. In the second and third strategies, we analyzed the entire PICU stay and adjusted for centre (the 7 participating sites were considered a possible confounding variable). The statistical analyses are described in detail in Appendix 1 (available at www.cmaj.ca/cgi/content/full /cmaj.081715/DC1).

\section{Results}

The median age of the 1806 patients was 24 months (IQR 590). Characteristics of the study population are given in Table 1. The median and mean PELOD scores differed between the seven PICUs (median range 10-11, mean range 8-14; both $p<0.001$ ).

Daily PELOD scores were measured on a total of 10361 days. The median and mean values for days 1 to 7 , day 14 and day 21 among survivors and nonsurvivors are given in Table 2 . The maximum daily score occurred on average on day 3 (median day 2 [IQR 1-3]). The maximum daily score differed between nonsurvivors and survivors (nonsurvivors: mean 8.6, median 2 [IQR 1-7] v. survivors: mean 2.6, median 2 [IQR 1-3]; $p<0.001)$. 
Of the 499 children without any organ dysfunction on day 1 , $113(22.6 \%)$ had dysfunction of one or more organs during their PICU stay; the mortality was $4.4 \%$ among these children, as compared with $0.2 \%$ among the 386 who did not have organ dysfunction during their stay $(p=0.003)$. Among the 1042 patients without multiple organ dysfunction syndrome on day 1 , $135(13.0 \%)$ acquired the syndrome during their PICU stay; the mortality was $8.9 \%$ among these children, as compared with $0.6 \%$ among the 907 who did not acquire the syndrome during their stay ( $p<0.001)$. On day 1, multiple organ dysfunction syndrome was present in $764(42.3 \%)$ of the patients. The syndrome worsened during the PICU stay in $133(17.4 \%)$ and remained unchanged or improved in $631(82.6 \%)$; the mortality was $25.6 \%$ among those in whom it worsened and $10.1 \%$ among the other children; $p<0.001$ ). New or progressive multiple organ dysfunction syndrome was reported in 899 (48.9\%) of the patients.

\section{PELOD score on day 1 and mortality}

Logistic regression analysis showed that the PELOD score on day 1 was a significant prognostic factor (odds ratio [OR] per point $1.16,95 \%$ confidence interval [CI] 1.13-1.18, after adjustment for centre). The analysis of the distribution of the day 1 scores identified three groups of scores (low [ $<10$ points], medium [10-19 points] and high $[\geq 20$ points]), with cutoff values of 10 and 20 points associated with increasing mortality (Table 3 ). The OR for death among children with a medium PELOD score on day 1 was 4.2 (95\% CI 2.0-8.7); the OR among those with a high score on day 1 was 40.7 (95\% CI 20.3-81.4). These findings show that the PELOD score on day 1 was an important predictor of the patients' outcome.

\section{Changes in daily scores during the first four days}

Our analysis of trends in daily PELOD scores during the first four days in PICU are shown in Table 3 and in Table e 1 of Appendix 1 (available at www.cmaj.ca/cgi/content/full/cmaj.081715/DC1). After adjustment for the day 1 score, the mortality was 50\% (19/38) among children with a high score on day 1 when the score increased on day 2 pupillary dilatation.
(Table 3). Without adjustment for the day 1 score (owing to the great number of categories), the mortality was $50 \%$ (7/14) when there was an increase in score on day 2 and when the score increased from day 2 to day 4 ; the mortality was 7\% (49/745) in other situations $(p<0.001)$ (Appendix 1 , Table e1). Increasing daily PELOD scores during the first four days in the PICU indicated a poor prognosis (Appendix 1, Table e1).

\begin{tabular}{|c|c|c|c|c|}
\hline \multirow[b]{2}{*}{ Organ system and variable } & \multicolumn{4}{|c|}{ Points assigned } \\
\hline & 0 & 1 & 10 & 20 \\
\hline \multicolumn{5}{|l|}{ Neurologic* } \\
\hline Glasgow coma score & $\begin{array}{l}12-15 \\
\text { and }\end{array}$ & $7-11$ & $\begin{array}{l}4-6 \\
\text { or }\end{array}$ & 3 \\
\hline Pupillary reaction & Both reactive & & Both fixed & \\
\hline \multicolumn{5}{|l|}{ Cardiovascular } \\
\hline \multicolumn{5}{|l|}{ Heart rate, beats/min } \\
\hline$<12$ years & $\leq 195$ & & $>195$ & \\
\hline \multirow{2}{*}{$\geq 12$ years } & $\leq 150$ & & $>150$ & \\
\hline \multirow{2}{*}{\multicolumn{5}{|c|}{ Systolic blood pressure, $\mathrm{mm} \mathrm{Hg}$}} \\
\hline & & & & \\
\hline$<1$ mo & $>65$ & & $35-65$ & $<35$ \\
\hline$\geq 1 \mathrm{mo}-<1 \mathrm{yr}$ & $>75$ & & $35-75$ & $<35$ \\
\hline$\geq 1 \mathrm{yr}-<12 \mathrm{yr}$ & $>85$ & & $45-85$ & $<45$ \\
\hline$\geq 12 \mathrm{yr}$ & $>95$ & & $55-95$ & $<55$ \\
\hline \multicolumn{5}{|l|}{ Renal } \\
\hline \multicolumn{5}{|l|}{ Creatinine, $\mu \mathrm{mol} / \mathrm{L}(\mathrm{mg} / \mathrm{dL})$} \\
\hline$<7 d$ & $<140(<1.59)$ & & \multicolumn{2}{|l|}{$\geq 140(\geq 1.59)$} \\
\hline$\geq 7 \mathrm{~d}-<1 \mathrm{yr}$ & $<55(<0.62)$ & & \multicolumn{2}{|l|}{$\geq 55(\geq 0.62)$} \\
\hline$\geq 1 \mathrm{yr}-<12 \mathrm{yr}$ & $<100(<1.13)$ & & \multicolumn{2}{|l|}{$\geq 100(\geq 1.13)$} \\
\hline$\geq 12 \mathrm{yr}$ & $<140(<1.59)$ & & \multicolumn{2}{|l|}{$\geq 140(\geq 1.59)$} \\
\hline \multicolumn{5}{|l|}{ Respiratory } \\
\hline $\mathrm{PaO}_{2}: \mathrm{FiO}_{2}$ ratio, $\mathrm{mm} \mathrm{Hg}$ & $>70$ & & \multirow{4}{*}{$\begin{array}{c}\leq 70 \\
\text { or } \\
>90(>11.7)\end{array}$} & \\
\hline & and & & & \\
\hline $\mathrm{PaCO}_{2}, \mathrm{~mm} \mathrm{Hg}(\mathrm{kPa})$ & $\begin{array}{c}\leq 90(\leq 11.7) \\
\text { and }\end{array}$ & & & \\
\hline Mechanical ventilationt & No ventilation & Ventilation & & \\
\hline \multicolumn{5}{|l|}{ Hematologic } \\
\hline Leukocyte count, $\times 10^{9} / \mathrm{L}$ & $\begin{array}{l}\geq 4.5 \\
\text { and }\end{array}$ & $\begin{array}{l}1.5-4.4 \\
\text { or }\end{array}$ & \multirow[t]{2}{*}{$<1.5$} & \\
\hline Platelet count, $\times 10^{9} / \mathrm{L}$ & $\geq 35$ & $<35$ & & \\
\hline \multicolumn{5}{|c|}{ Hepatic } \\
\hline Glutamic oxaloacetic transaminase, IU/L & $\begin{array}{c}<950 \\
\text { and }\end{array}$ & $\begin{array}{l}\geq 950 \\
\text { or }\end{array}$ & & \\
\hline $\begin{array}{l}\text { Prothrombin time, \% of standard } \\
\text { (international normalized ratio) }\end{array}$ & $>60(<1.40)$ & $\leq 60(\geq 1.40)$ & & \\
\hline
\end{tabular}

Note: $\mathrm{FiO}_{2}=$ fraction of inspired oxygen, $\mathrm{PaCO}_{2}=$ partial pressure of carbon dioxide in arterial blood, $\mathrm{PaO}_{2}=$ partial pressure of oxygen in arterial blood.

*For the Glasgow coma score, use the lowest value. If the patient is sedated, record the estimated coma score before sedation. Assess the patient only with known or suspected acute central nervous system disease. For pupillary reactions, nonreactive pupils must be $>3 \mathrm{~mm}$; do not assess after iatrogenic

tThe use of mask ventilation is not considered to be mechanical ventilation.

Figure 2: Calculation of the daily PELOD (Pediatric Logistic Organ Dysfunction) score. Each organ dysfunction receives points for the variable associated with the highest points. For example, if the worst heart rate of the day was 200 beats/min (10 points) and the systolic blood pressure remained at $30 \mathrm{~mm} \mathrm{Hg}$ (20 points), then 20 points is assigned. When a variable is measured more than once in the 24 hours, the most severe value is used in calculating the score. The maximum number of points for an organ is 20 , and the maximum daily PELOD score is 71 . Adapted, with permission, from Leteurtre et $a^{9}$ (Copyright ( 1999 SAGE Publications). 


\section{Mean rate of change in PELOD score during the entire PICU stay}

The changes in daily PELOD score over time in the three score groups (low, medium and high day 1 scores) differed between survivors and nonsurvivors. In all three groups, the difference in daily score between survivors and nonsurvivors increased over time (see Figure e1 in Appendix 1, available at www.cmaj.ca/cgi/content/full/cmaj.081715/DC1). In the group of patients with a low PELOD score on day $1(<10$

Table 1: Characteristics of 1806 critically ill children admitted to pediatric intensive care units

\begin{tabular}{|c|c|}
\hline Characteristic & $\begin{array}{c}\text { No. }(\%) \text { of } \\
\text { patients* } \\
n=1806\end{array}$ \\
\hline Age, mo, median (IQR) & $24(5-90)$ \\
\hline \multicolumn{2}{|l|}{ Age group } \\
\hline$<28$ d (neonates) & 171 (9.5) \\
\hline 1 mo- $<1$ yr (infants) & $525(29.1)$ \\
\hline $1-<12$ yr (children) & $853(47.2)$ \\
\hline$\geq 12$ yr (adolescents) & $257(14.2)$ \\
\hline Male:female ratio & 1.2 \\
\hline Surgical patients & $882(48.8)$ \\
\hline Mechanical ventilation required & $921(51.0)$ \\
\hline PRISM score, median (IQR) & $6(2-10)$ \\
\hline \multicolumn{2}{|l|}{$\begin{array}{l}\text { Organ system of primary dysfunction } \\
\text { on admission }\end{array}$} \\
\hline Cardiovascular & $485(26.9)$ \\
\hline Neurologic & $335(18.6)$ \\
\hline Respiratory & $631(34.9)$ \\
\hline Hepatic & 34 (1.9) \\
\hline Genitourinary & 35 (1.9) \\
\hline Gastrointestinal & $91 \quad(5.0)$ \\
\hline Endocrine & $22(1.2)$ \\
\hline Musculoskeletal & 68 (3.8) \\
\hline Hematologic & $24 \quad(1.3)$ \\
\hline Other/undetermined & $81 \quad(4.5)$ \\
\hline \multicolumn{2}{|l|}{ Primary category of illness on admission } \\
\hline Infection & $439(24.3)$ \\
\hline Trauma & 175 (9.7) \\
\hline Congenital disease & $663(36.7)$ \\
\hline Chemical injury & $25 \quad(1.4)$ \\
\hline Drug & $12(0.7)$ \\
\hline Cancer & $60 \quad(3.3)$ \\
\hline Diabetes & $18 \quad(1.0)$ \\
\hline Allergic or immunologic disease & $42 \quad(2.3)$ \\
\hline Other/undetermined & $372(20.6)$ \\
\hline No. who died in PICU & 115 (6.4) \\
\hline Length of stay in PICU, d, median (IQR) & $3(2-6)$ \\
\hline
\end{tabular}

Note: IQR = interquartile range, $\mathrm{PICU}=$ pediatric intensive care unit, PRISM = pediatric risk of mortality.

*Unless stated otherwise. points), the mean rate of change in daily score was 0 points per day among survivors and 0.3 points per day among nonsurvivors $(p<0.001)$. The corresponding mean rate of change among patients with a medium score on day 1 (10-19 points) was -0.5 points per day among survivors and 0 points per day among nonsurvivors $(p<0.001)$; among those with a high score on day 1 ( $\geq 20$ points), it was -0.9 points per day among survivors and -0.5 points per day among nonsurvivors $(p<0.001)$. These findings show that even small increases in the PELOD score and a lack of improvement in score were worrisome signs.

\section{Optimal days for measuring daily scores}

Graphic distributions of the median PELOD scores over time are shown in Figures e2 and e3 in Appendix 1 (available at www.cmaj.ca/cgi/content/full/cmaj.081715/DC1). A set of seven days was found to be associated with the highest hazard ratios for death: days 1, 2, 5, 8, 12, 16 and 18 (Figure 3).

\section{Interpretation}

We found that the PELOD score on day 1 was a significant prognostic factor of death. In the three groups of patients stratified by low ( $<10$ points), medium (10-19 points) and high ( $\geq 20$ points) PELOD score on day 1 , the change in the severity of organ dysfunction over time differed between survivors and nonsurvivors: not just an increasing score, but also a score showing no improvement, was an adverse prognostic factor. Moreover, regardless of what the initial PELOD score was on day 1 , an increase in the score from day 1 to day 2 and from day 2 to day 4 was associated with a mortality of $50 \%$. Finally, we found that a set of seven days for daily measurement of the PELOD score provided optimal information on the progression of multiple organ dysfunction syndrome during the stay in the PICU.

Analyses of the trends of multiple organ dysfunction syndrome over time have been performed with several method-

Table 2: Daily PELOD scores among critically ill children admitted to pediatric intensive care units*

\begin{tabular}{cccccc}
\hline & \multicolumn{4}{c}{ Patients; PELOD score } \\
\cline { 2 - 3 } \cline { 5 - 6 } Day & \multicolumn{2}{c}{ Nonsurvivors } & & \multicolumn{2}{c}{ Survivors } \\
\cline { 2 - 3 } \cline { 5 - 6 } & Mean & Median (IQR) & & Mean & Median (IQR) \\
\hline 1 & $22.7^{*}$ & $21(11-31)$ & & 6.9 & $2(0-11)$ \\
\hline 2 & $20.3^{*}$ & $21(11-31)$ & & 6.2 & $2(0-11)$ \\
\hline 3 & $16.8^{*}$ & $13(10-22)$ & & 6.1 & $1(0-11)$ \\
\hline 4 & $15.6^{*}$ & $12(6-21)$ & & 5.7 & $1(0-11)$ \\
\hline 5 & $14.5^{*}$ & $12(10-21)$ & & 5.4 & $1(0-10)$ \\
\hline 7 & $14.9^{*}$ & $11(10-22)$ & & 5.5 & $1(0-11)$ \\
\hline 14 & $13.1^{*}$ & $11(1-21)$ & & 5.6 & $1(0-11)$ \\
\hline 21 & $11.1^{*}$ & $11(1-21)$ & & 5.5 & $1(0-10)$ \\
\hline
\end{tabular}

Note: IQR = interquartile range, PELOD score = Pediatric Logistic Organ Dysfunction score.

${ }^{*} p<0.001$, Wilcoxon two-sample test (survivors v. nonsurvivors). 
ologic strategies and different times of data collection in adult patients. ${ }^{10,14-17,19,20}$ Many studies have shown that daily monitoring of organ dysfunction can be useful for estimating the response to therapy. ${ }^{21-23}$ We believe that the model proposed in our study - to measure the PELOD score on days 1, 2, 5, 8, 12,16 and 18 - provides the best balance between the workload of assessing daily scores and the optimal association with prognosis throughout the PICU stay. Findings from studies involving critically ill adults support such an approach. In a study by Timsit and colleagues, the severity of organ dysfunction on any given day during the first week in ICU was an important predictor of in-hospital mortality. ${ }^{24}$ In a study by Wagner and associates, the acute physiology score on a day in the first week in ICU contributed to 54\% of the prediction of in-hospital mortality, as compared with only $5 \%$ for the acute physiology score at admission. ${ }^{25}$ Moreover, in studies involving patients with long ICU stays, severity scores at admission failed to predict mortality. ${ }^{24,26}$ The late events could not be predicted with scores at admission or on the first day. This suggests that, for patients with prolonged stays in the ICU, the calculation of scores on later days may be more useful.

Rates of death have been reported to be higher among critically ill adults $(>20 \%)^{17,27}$ than among critically ill children $(4 \%-5 \%))^{8,28}$ This may explain why a mortality of at least $50 \%$ was associated with an increase in the Sequential Organ Failure Assessment score among adult patients during the first two days in $\mathrm{ICU}^{17}$ and, in our study, with an increase in the PELOD score during the first four days in PICU (occurring in only $1.8 \%$ of our sample). Because the length of stay in a PICU is usually short (median three days in our study), we converted time into discrete day intervals to determine the ideal sequence of measurement of daily PELOD scores. Cook and associates converted time into discrete week intervals. ${ }^{15}$

Measurement of the severity of multiple organ dysfunction syndrome is needed to describe the clinical course of groups of critically ill children. ${ }^{13}$ Our study showed that the progression of the daily PELOD score provided prognostic information in addition to the highest PELOD score for the entire PICU stay. These data support the concept that the PELOD score and its progression in the PICU can be an outcome measure of interest. Measurement of organ dysfunc- tion with scores such as the PELOD can be useful both for administrative PICU management ${ }^{29-31}$ and as a secondary outcome measure of studies conducted in the ICU, ${ }^{12,32}$ particularly the PICU, ${ }^{21,33}$ where the death rate is low. Two recent examples can be given. Among critically ill children, a restrictive transfusion strategy with a hemoglobin threshold of $7 \mathrm{~g} / \mathrm{dL}$, compared with a liberal transfusion strategy, resulted in a $96 \%$ decrease in the number of patients who had any transfusion exposure and a $44 \%$ decrease in the number of units of red blood cells transfused; these results were not associated with increased rates of new or progressive multiple organ dysfunction syndrome or increasing daily PELOD scores. ${ }^{21}$

The second example concerns the H1N1 influenza pandemic. With scarce resources, the change in PELOD score over several days may be of help to policy-makers faced the decision of triaging care between patients with severe H1N1 influenza. In such circumstances, medical policy can be informed by PELOD scores. Similar use of the Sequential Organ Failure Assessment score as an initial, 48-hour and 120-hour triage tool for critical care of adults during an influenza pandemic has been proposed..$^{29,34}$

\section{Strengths and limitations}

The strengths of our study was that it was prospective and conducted in seven PICUs over three countries. Also, we included a large number of critically ill children whose combined stay in the PICUs was more than 10000 consecutive days. We used three different statistical methods to analyze the changes in daily PELOD score, all of which gave consistent results.

Our study is not without limitations. First, we monitored only mortality in the PICU and have no data for patients discharged from the PICU. However, the number of children who die in hospital after discharge from the PICU is small $\left(0.9 \%\right.$ in the study by Kanter and colleagues $\left.{ }^{35}\right)$. Second, the number of deaths among the 338 patients who stayed in the PICU longer than seven days was low $(n=33)$. The validity of the daily PELOD scores after one week in the PICU (on days $8,12,16$ and 18) should be validated in future prospective studies involving more critically ill children with longterm stays.

Table 3: Mortality associated with PELOD score on day 1 and change in score from day 1 to day 2

\begin{tabular}{|c|c|c|c|c|c|c|}
\hline $\begin{array}{l}\text { Change in score } \\
\text { from day } 1 \text { to day } 2\end{array}$ & \multicolumn{6}{|c|}{ PELOD score on day 1} \\
\hline Baseline (day 1) & $10 / 887$ & $1.1 *(0.5-2.1)$ & $31 / 660$ & $4.7(3.2-6.6)$ & $74 / 259$ & $28.6(23.2-34.5)$ \\
\hline Decrease & $1 / 122$ & $0.8 \quad(0.0-4.5)$ & $8 / 245$ & $3.3(1.4-6.3)$ & $16 / 126$ & $12.7 \quad(7.4-19.8)$ \\
\hline
\end{tabular}

Note: $\mathrm{Cl}=$ confidence interval, PELOD score = Pediatric Logistic Organ Dysfunction score.

*For the 10 patients with a low PELOD score on day 1 who died, the underlying disease on admission was chronic cardiac disease ( $n=7$ ), chronic respiratory disease $(n=1)$, immunodeficiency $(n=1)$ and acute hepatic failure $(n=1)$. 


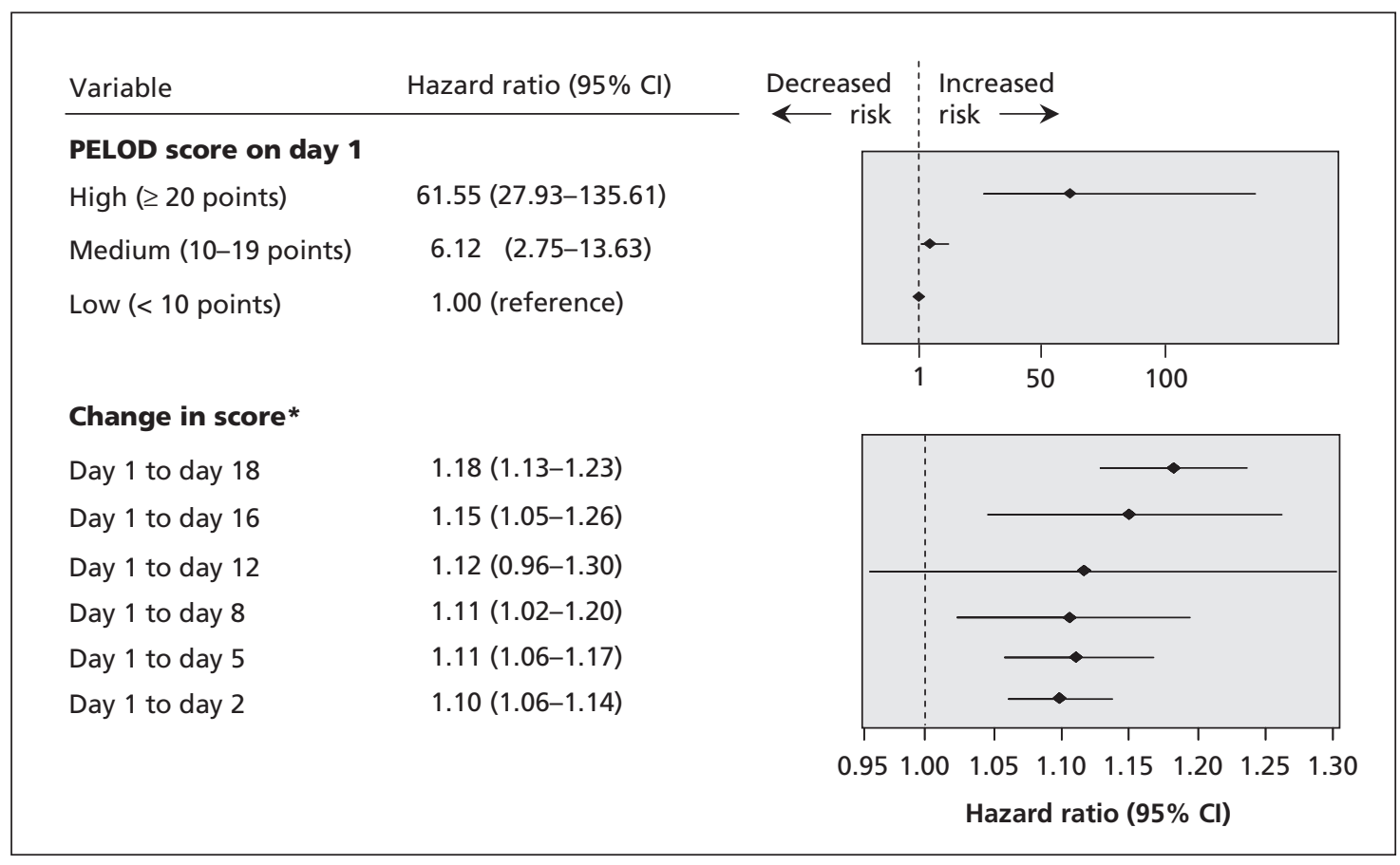

Figure 3: Serial evaluation of the change in the daily PELOD (Pediatric Logistic Organ Dysfunction) score from day 1, adjusted for baseline value (PELOD score on day 1). *Hazard ratio (HR) of death and $95 \%$ confidence interval $(\mathrm{Cl})$ are reported per point increase in the change in score. The cumulative HR of death was calculated as follows: (HR of PELOD score on day 1$) \times(H R$ of change in score from day 1 to specified day). For example, for a child whose score is 23 on day 1 and 13 on day 5 , the change in score is -10 ; the HR for death would be 21.48 $\left[61.55 \times\left(1.11^{-10}\right)=61.55 \times 0.35\right]$. For a child whose score is 12 on day 1 and 32 on day 16 , the change in score is 20 ; in this instance, the HR for death would be $98.37\left[6.12 \times\left(1.15^{20}\right)=6.12 \times 16.08\right]$.

\section{Conclusion}

Measuring changes in organ dysfunction over time is important. First, a daily PELOD score that shows a worsening condition or no improvement over time is a strong prognostic factor for death. This information may be helpful at the bedside, particularly within the first four days in the patient's course. Second, changes in the PELOD score describe the patterns and trajectories of multiple organ dysfunction over time. Such changes can also be used as a marker of severity of illness in clinical studies. We found that a set of seven days for the measurement of daily PELOD scores provided optimal information on the progression of multiple organ dysfunction syndrome during the stay in the PICU. This information could be used for epidemiologic and administrative purposes.

This article has been peer reviewed.

Competing interests: None declared.

Contributors: Stéphane Leteurtre, Francis Leclerc and Bruno Grandbastien contributed to the study concept and design. Stéphane Leteurtre, François Proulx, Jacques Cotting, Ronald Gottesman, Ari Joffe, Bendicht Wagner and Philippe Hubert contributed to the acquisition of the data. Stéphane Leteurtre, Alain Duhamel, Bruno Grandbastien, Jacques Lacroix, Francis Leclerc and Alain Martinot contributed to the analysis and interpretation of the data. Stéphane Leteurtre, Francis Leclerc, Jacques Lacroix and Alain Duhamel drafted the manuscript. All of the authors critically revised the manuscript for important intellectual content and approved the final version submitted for publication.
Acknowledgement: These data were presented in part at the 5th World Congress on Pediatric Critical Care, June 24-28, 2007, in Geneva, Switzerland.

Funding: This study was supported by the Programme Hospitalier de Recherche Clinique 1997 of the French Health Ministry. The funders had no role in the design or conduct of the study, the collection, management, analysis or interpretation of the data, or the preparation, review or approval of the manuscript. The researchers are independent from the funders.

\section{REFERENCES}

1. Cengiz P, Zimmerman JJ. Prelude to pediatric multiple organ dysfunction syndrome: the golden hours concept revisited. Pediatr Crit Care Med 2003;4:263-4.

2. Marshall JC. Modeling MODS: What can be learned from animal models of the multiple-organ dysfunction syndrome? Intensive Care Med 2005;31:605-8.

3. Proulx F, Gauthier M, Nadeau D, et al. Timing and predictors of death in pediatric patients with multiple organ system failure. Crit Care Med 1994;22:1025-31.

4. Wilkinson JD, Pollack MM, Glass NL, et al. Mortality associated with multiple organ system failure and sepsis in pediatric intensive care unit. J Pediatr 1987;111: 324-8.

5. Tantalean JA, Leon RJ, Santos AA, et al. Multiple organ dysfunction syndrome in children. Pediatr Crit Care Med 2003;4:181-5.

6. Proulx F, Joyal JS, Mariscalco MM, et al. The pediatric multiple organ dysfunction syndrome. Pediatr Crit Care Med 2009;10:12-22.

7. Proulx F, Fayon M, Farrell CA, et al. Epidemiology of sepsis and multiple organ dysfunction syndrome in children. Chest 1996;109:1033-7.

8. Graciano AL, Balko JA, Rahn DS, et al. The Pediatric Multiple Organ Dysfunction Score (P-MODS): development and validation of an objective scale to measure the severity of multiple organ dysfunction in critically ill children. Crit Care Med 2005; 33:1484-91.

9. Leteurtre S, Martinot A, Duhamel A, et al. Development of a pediatric multiple organ dysfunction score: use of two strategies. Med Decis Making 1999;19:399-410.

10. Leteurtre S, Martinot A, Duhamel A, et al. Validation of the paediatric logistic organ dysfunction (PELOD) score: prospective, observational, multicentre study. Lancet 2003;362:192-7.

11. Marshall JC. Organ dysfunction as an outcome measure in clinical trials. Eur J Surg Suppl 1999;584:62-7.

12. Petros AJ, Marshall JC, van Saene HK. Should morbidity replace mortality as an 
endpoint for clinical trials in intensive care? Lancet 1995:345:369-71.

13. Lacroix J, Cotting J. Severity of illness and organ dysfunction scoring in children. Pediatr Crit Care Med 2005;6:S126-34

14. Cappi SB, Sakr Y, Vincent JL. Daily evaluation of organ function during renal replacement therapy in intensive care unit patients with acute renal failure. J Crit Care 2006;21:179-83.

15. Cook R, Cook D, Tilley J, et al. Multiple organ dysfunction: baseline and serial component scores. Crit Care Med 2001;29:2046-50.

16. Doig CJ, Zygun DA, Fick GH, et al. Study of clinical course of organ dysfunction in intensive care. Crit Care Med 2004;32:384-90.

17. Ferreira FL, Bota DP, Bross A, et al. Serial evaluation of the SOFA score to predict outcome in critically ill patients. JAMA 2001;286:1754-8.

18. Briskin A, Hladunewich M. What's my mother's SOFA today, doc? Commentary on: daily evaluation of organ function during renal replacement therapy in ICU patients with acute renal failure. J Crit Care 2006;21:183-4.

19. Kilic YA, Yorganci K, Sayek I. Visualizing multiple organ failure: a method for analyzing temporal and dynamic relations between failing systems and interventions. Crit Care 2007;11:417

20. Moreno R, Vincent JL, Matos R, et al. The use of maximum SOFA score to quantify organ dysfunction/failure in intensive care. Results of a prospective, multicentre study. Working Group on Sepsis related Problems of the ESICM. Intensive Care Med 1999;25:686-96.

21. Lacroix J, Hebert PC, Hutchison JS, et al. Transfusion strategies for patients in pediatric intensive care units. N Engl J Med 2007;356:1609-19.

22. Rivera-Fernandez R, Nap R, Vazquez-Mata G, et al. Analysis of physiologic alterations in intensive care unit patients and their relationship with mortality. $J$ Crit Care 2007;22:120-8.

23. Vincent JL, Baron JF, Reinhart K, et al. Anemia and blood transfusion in critically ill patients. JAMA 2002;288:1499-507.

24. Timsit JF, Fosse JP, Troche G, et al. Calibration and discrimination by daily Logistic Organ Dysfunction scoring comparatively with daily Sequential Organ Failure Assessment scoring for predicting hospital mortality in critically ill patients. Crit Care Med 2002;30:2003-13.

25. Wagner DP, Knaus WA, Harrell FE, et al. Daily prognostic estimates for critically ill adults in intensive care units: results from a prospective, multicenter, inception cohort analysis. Crit Care Med 1994:22:1359-72.

26. Ferraris VA, Propp ME. Outcome in critical care patients: a multivariate study. Crit Care Med 1992;20:967-76.

27. Vincent JL, de Mendonca A, Cantraine F, et al. Use of the SOFA score to asses the incidence of organ dysfunction/failure in intensive care units: results of a multicenter, prospective study. Working group on "sepsis-related problems" of the European Society of Intensive Care Medicine. Crit Care Med 1998;26: $1793-800$

28. Slater A, Shann F. The suitability of the Pediatric Index of Mortality (PIM), PIM2, the Pediatric Risk of Mortality (PRISM), and PRISM III for monitoring the quality of pediatric intensive care in Australia and New Zealand. Pediatr Crit Care Med 2004;5:447-54.

29. Christian MD, Hawryluck L, Wax RS, et al. Development of a triage protocol for critical care during an influenza pandemic. CMAJ 2006;175:1377-81.

30. Vincent JL, Ferreira FL. Evaluation of organ failure: we are making progress Intensive Care Med 2000;26:1023-4.

31. Welke KF, Karamlou T, Diggs BS. Databases for assessing the outcomes of the treatment of patients with congenital and paediatric cardiac disease - a comparison of administrative and clinical data. Cardiol Young 2008;18(Suppl 2):137-44.

32. Marshall JC. Measuring organ dysfunction in the intensive care unit: Why and how? Can J Anaesth 2005;52:224-30.

33. Nguyen TC, Han YY, Kiss JE, et al. Intensive plasma exchange increases a disintegrin and metalloprotease with thrombospondin motifs-13 activity and reverses organ dysfunction in children with thrombocytopenia-associated multiple organ failure. Crit Care Med 2008;36:2878-87.

34. Hebert PC, MacDonald N. Preparing for pandemic (H1N1) 2009. CMAJ 2009;181: E102-5.

35. Kanter RK. Post-intensive care unit pediatric hospital stay and estimated costs. Cri Care Med 2000;28:220-3.

Correspondence to: Prof. Francis Leclerc, Réanimation pédiatrique, Hôpital Jeanne de Flandre, 2 ave Eugène Avinée, 59037 Lille Cedex, France; francis.leclerc@chru-lille.fr

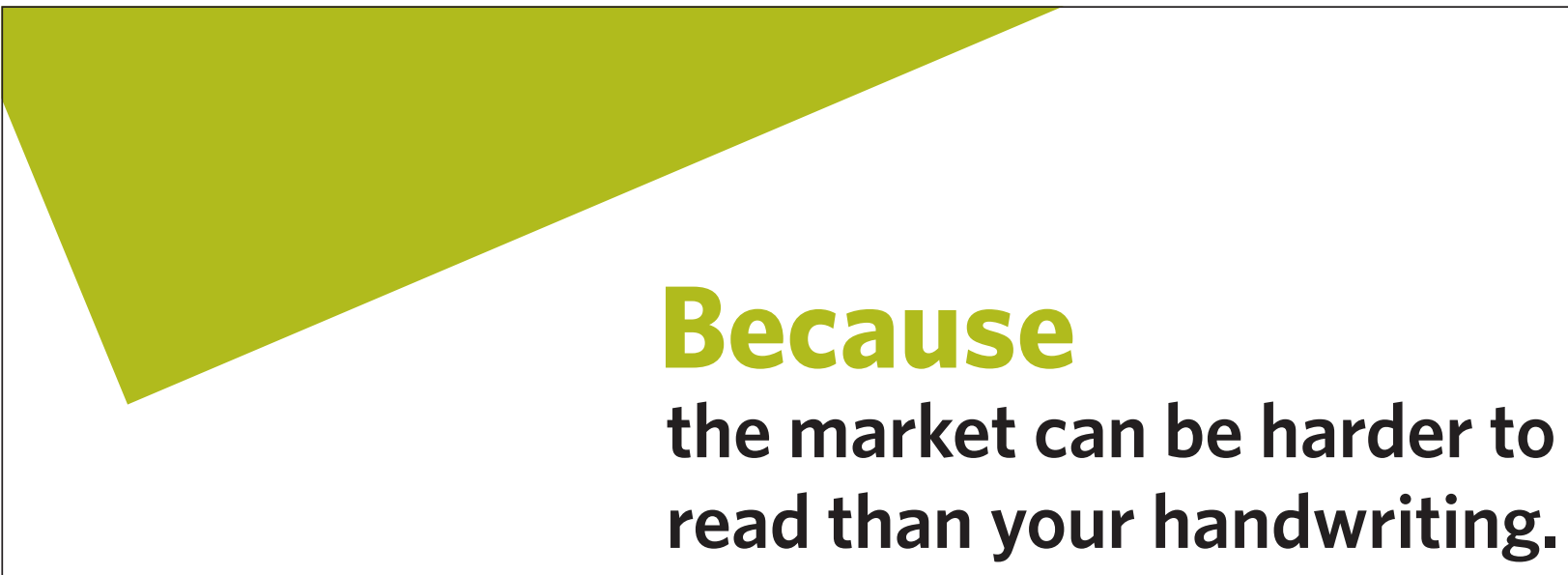

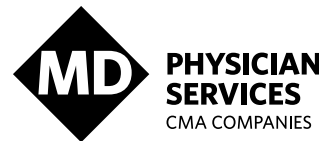

CMA COMPANIES

Financial | Practice Living
With guidance from experts who focus solely on wealth management for physicians, you can make sure your investments are working as hard as they should be. Let us show you how an intelligent, integrated approach to wealth management can help you invest to achieve your goals.

\section{Physician Services. Specializing in you.}

Find out how our solutions can transform your finances at md.cma.ca/because | 1877 877-3707 